\title{
Cephalometric Evaluation of Alveolar Bone Remodeling following Anterior Teeth Retraction
}

\author{
${ }^{1}$ Darshit Dhanani, ${ }^{2} \mathrm{G}$ Shivaprakash
}

\section{ABSTRACT}

Aim: To evaluate the extent of the alveolar bone remodeling after incisor retraction using lateral cephalograms.

Materials and methods: Lateral cephalograms of 30 patients with age of 16 years and above requiring therapeutic extraction of both maxillary and mandibular first premolars, mainly for the purpose of retraction of anterior teeth, had been taken at the start of treatment and after retraction of anterior teeth. Various hard tissue anatomical landmarks were traced, and linear parameters of pretreatment (T1) and postretraction (T2) lateral cephalometric radiographs were measured. The mean and standard deviation were calculated, the data were tabulated, and comparison of T1 and T2 readings was made utilizing paired Student's t-test.

Results: When maxillary incisors are retracted, the labial bone thickness at the midroot level (MxL2) and at apical level (MxL3) increased during upper incisor retraction. There was a significant reduction in alveolar bone thickness on the lingual/palatal side after maxillary and mandibular incisor retraction.

Conclusion: When tooth movement is limited, forcing the tooth against the cortical bone may cause adverse sequelae. This type of approach must be carefully monitored to avoid negative iatrogenic effects.

Keywords: Alveolar bone remodeling, Incisor retraction, Labial bone thickness, Lateral cephalograms.

How to cite this article: Dhanani D, Shivaprakash G. Cephalometric Evaluation of Alveolar Bone Remodeling following Anterior Teeth Retraction. CODS J Dent 2016;8(1):21-24.

Source of support: Nil

Conflicts of interest: None

\section{INTRODUCTION}

Orthodontic tooth movement is a process whereby the application of a force induces bone resorption on the pressure side and bone apposition on the tension side. Controversy exists whether the changes occurring in anterior alveolar bone during orthodontic tooth movement always follow the direction and extent of tooth movement.

\footnotetext{
${ }^{1}$ Postgraduate Student, ${ }^{2}$ Professor and Head

1,2Department of Orthodontics and Dentofacial Orthopedics College of Dental Sciences, Davangere, Karnataka, India

Corresponding Author: Darshit Dhanani, Postgraduate Student, Department of Orthodontics and Dentofacial Orthopedics College of Dental Sciences, Davangere, Karnataka India, e-mail: dhananidarshit41@gmail.com
}

A basic axiom in orthodontics is "bone traces tooth movement," which suggests that whenever orthodontic tooth movement occurs, bone around the alveolar socket will remodel to the same extent, i.e., a ratio of bone remodeling to tooth movement (B/T) of 1:1 develops. However, nonorthodontic tooth movements do not show coherence with this rule. ${ }^{1}$

Our ability to move teeth within the limits of the alveolar trough is confirmed daily in orthodontic practice with bodily retraction of canines in premolar extraction cases. Less clear, however, is our ability to bodily retract incisors over similar distances. Unlimited tooth movement assumes that the osseous housing can fully reconstitute itself in any direction the tooth is moved. However, clinical experience indicates that some limitations to anteriorposterior incisor movement are operative. ${ }^{2}$

The dimension of the anterior alveolus appears to set limits to orthodontic treatment, and challenging these boundaries may accelerate iatrogenic sequelae. Therefore, a study was carried out to evaluate the extent of the alveolar bone remodeling after incisor retraction.

\section{MATERIALS AND METHODS}

The subjects comprised of 30 patients, all requiring first premolar extraction as therapeutic procedure. The subjects selected were gathered from the records of Department of Orthodontics, College of Dental Sciences, Davangere, India.

\section{Inclusion Criteria}

- Patients with age 16 years

- Therapeutic extraction of maxillary and mandibular first premolars

- Malocclusion with minimum crowding ( $\leq 3 \mathrm{~mm})$

- Overjet $\geq 5 \mathrm{~mm}$

- All the cases were treated by preadjusted edgewise appliance mechanotherapy using MBT prescription $0.022^{\prime \prime}$ slot

\section{Exclusion Criteria}

- Patient with age $\geq 25$ years

- Patients with gross facial asymmetry

- Patients suffering from any craniofacial syndromes

- Patients having any other skeletal deformity 


\section{METHOD OF STUDY}

Lateral cephalograms of patients with an age of 16 years and above had been taken at the start of treatment and after retraction of anterior teeth. All the linear measurements of pretreatment (T1) and postretraction (T2) lateral cephalometric radiographs were measured. The mean and standard deviation (SD) were calculated, the data were tabulated, and comparison of $\mathrm{T} 1$ and $\mathrm{T} 2$ readings was made utilizing paired Student's t-test for statistical significance between the two sets of measurements.

For alveolar bone remodeling, the thickness of the labial and palatal (lingual) alveolar plates was measured at the site adjacent to the widest point of the labiopalatal root in three slices separated by $3 \mathrm{~mm}$ (S1, S2, and S3 respectively) (Fig. 1). At the beginning of treatment, six measurements were taken for maxillary incisor and six measurements were taken for mandibular incisor; three on the labial side and three on the lingual side (T1). The same measurements were repeated after incisor retraction was completed (T2).

\section{For Maxillary Teeth}

- MxL1 - distance from the cervical third of labial root surface to cortical plate

- MxL2 - distance from the middle third of labial root surface to cortical plate

- MxL3 - distance from the apical third of labial root surface to cortical plate

- MxP1 - distance from the cervical third of palatal root surface to cortical plate

- MxP2 - distance from the middle third of palatal root surface to cortical plate

- $\mathrm{MxP3}$ - distance from the apical third of palatal root surface to cortical plate.

\section{For Mandibular Teeth}

- MnL1 - distance from the cervical third of labial root surface to cortical plate

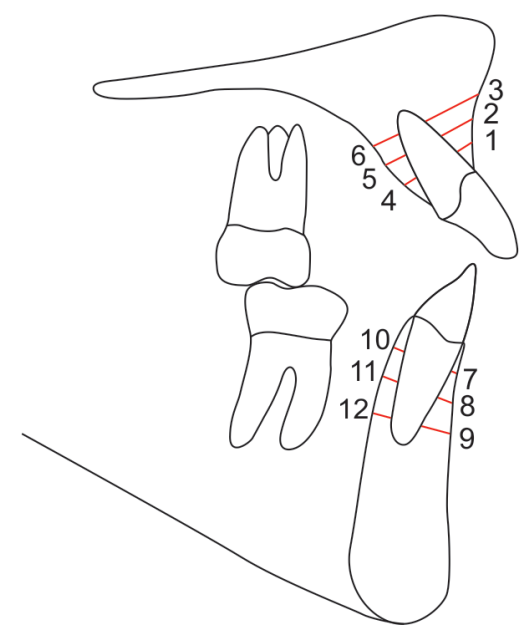

Fig. 1: Linear measurements of alveolar bone remodeling
- MnL2 - distance from the middle third of labial root surface to cortical plate

- MnL3 - distance from the apical third of labial root surface to cortical plate

- MnLg1 - distance from the cervical third of lingual root surface to cortical plate

- MnLg2 - distance from the middle third of lingual root surface to cortical plate

- MnLg3-distance from the apical third of lingual root surface to cortical plate.

\section{Statistical Analysis}

All the data were analyzed using Statistical Package for the Social Sciences software version 20 (SPSS Inc; Chicago, Illinois, USA). Results were presented as mean \pm SD. The results thus obtained will be statistically analyzed using paired Student's t-test to compare T1 and T2 values. The significance level of $p<0.05$ was selected.

\section{RESULTS}

The change in maxillary labial bone thickness was not statistically significant except the width of the bone labial to the maxillary incisor decreased significantly at MxL1 $(\mathrm{p}<0.001)$.

Regarding the maxillary bone thickness lingual to the incisors, $\mathrm{MxP} 3$ measurements showed minimal change, but the measurements at the MxP1 and MxP2 levels differed significantly over time. The changes were significant at the coronal level $(\mathrm{MxP} 1)(\mathrm{p}<0.001)$. Alveolar bone width at the midroot level (MxP2) also decreased significantly $(\mathrm{p}<0.001)$. At the apical level $(\mathrm{MxP} 3)$, changes were significant $(\mathrm{p}<0.01)$.

Teeth decreased in thickness at the coronal level (MnL1), midroot level (MnL2), and apical level (MnL3) $(\mathrm{p}<0.001)$.

The lingual alveolar bone of the mandible decreased significantly after retraction of the incisors. The decrease was significant at coronal level (MnLg1), mid-root level (MnLg2), and apical level (MnLg3) $(\mathrm{p}<0.001$; Table 1 and Graph 1).

\section{DISCUSSION}

The anatomical limits set by the cortical plates of the alveolus may be regarded as orthodontic walls. ${ }^{2}$ Unlimited tooth movement is not possible during retraction of the incisors, especially the mandibular incisors. This is due to the restriction imposed by the symphyseal bone. It consists of a dense cortical plate on the labial and lingual surfaces near the roots of the incisors.

The present study is carried out to evaluate the changes in labial and lingual alveolar bone during 
Table 1: Means and SD of maxillary and mandibular alveolar bone width measured on labial and lingual side before and after retraction of anterior teeth

\begin{tabular}{|c|c|c|c|c|c|c|c|c|c|}
\hline \multirow[b]{2}{*}{ Variables } & \multicolumn{2}{|c|}{ Pretreatment (T1) } & \multicolumn{2}{|c|}{ Postretraction (T2) } & \multicolumn{2}{|c|}{ Changes } & \multirow[b]{2}{*}{ Minimum } & \multirow[b]{2}{*}{ Maximum } & \multirow[b]{2}{*}{$p$-value } \\
\hline & Mean & $S D$ & Mean & $S D$ & Mean & $S D$ & & & \\
\hline MxL1 & 1.07 & 0.52 & 0.60 & 0.38 & 0.47 & 0.32 & 0.00 & 1.00 & $0^{* \star \star}$ \\
\hline MxL2 & 1.52 & 0.68 & 1.57 & 0.54 & -0.05 & 0.50 & -1.00 & 1.00 & 0.586 \\
\hline MxL3 & 2.42 & 0.92 & 2.50 & 0.80 & -0.08 & 0.56 & -1.00 & 1.00 & 0.420 \\
\hline MxP1 & 1.50 & 0.53 & 0.72 & 0.45 & 0.78 & 0.58 & -1.00 & 2.00 & $0^{* * *}$ \\
\hline MxP2 & 2.17 & 0.71 & 1.55 & 0.99 & 0.62 & 1.12 & -2.00 & 2.50 & $0^{* * *}$ \\
\hline MxP3 & 2.95 & 0.87 & 2.27 & 1.27 & 0.68 & 1.29 & -2.00 & 3.00 & $0.007^{\star *}$ \\
\hline MnL1 & 0.73 & 0.37 & 0.42 & 0.35 & 0.32 & 0.36 & 0.00 & 1.00 & $0^{* * \star}$ \\
\hline MnL2 & 1.37 & 0.47 & 0.90 & 0.38 & 0.47 & 0.43 & -0.50 & 1.00 & $0^{* * *}$ \\
\hline MnL3 & 2.23 & 0.84 & 1.72 & 0.54 & 0.52 & 0.44 & -0.50 & 1.50 & $0^{\star \star *}$ \\
\hline MnLg1 & 1.03 & 0.39 & 0.60 & 0.38 & 0.43 & 0.34 & 0.00 & 1.00 & $0^{\star \star \star}$ \\
\hline MnLg2 & 1.63 & 0.49 & 1.08 & 0.40 & 0.55 & 0.30 & 0.00 & 1.50 & $0^{* * *}$ \\
\hline MnLg3 & 2.28 & 0.61 & 1.65 & 0.53 & 0.63 & 0.41 & 0.00 & 1.50 & $0^{* * *}$ \\
\hline
\end{tabular}

$\mathrm{p}<0.05 ;{ }^{* *} \mathrm{p}<0.01 ;{ }^{* *} \mathrm{p}<0.001 ;$ SD: Standard deviation

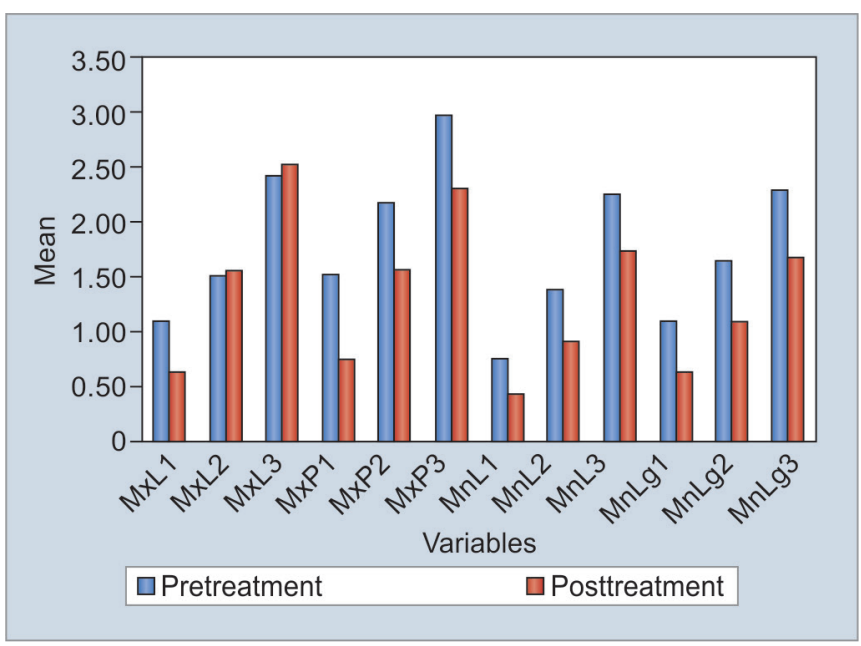

Graph 1: Means of maxillary and mandibular alveolar bone width measured before and after retraction of anterior teeth

incisor retraction in the maxilla and mandible. Based on the assumption that maximum tooth movement would challenge anatomical limits, the present study was undertaken to evaluate the extent of the alveolar bone remodeling after incisor retraction.

The results demonstrate that lingual movements of the maxillary and mandibular incisors reduced the lingual alveolar bone in both arches. This finding disputes that of DeAngelis, ${ }^{3}$ who presented the bending capacity of alveolar bone. According to DeAngelis, ${ }^{3}$ mechanotherapy induces alveolar distortion, and the distorted alveolus alters the electric environment, a process, i.e., attributed to the piezoelectricity of bone. As a result, the theory is that highly synchronized coordinated changes are triggered and with coordinated apposition and resorption, the alveolar bone retains its structural characteristics and size as it moves. In our patients, the maxillary and mandibular alveolar bone thickness did not remain the same; rather, it decreased. This finding of reduced alveolar bone thickness in the direction of tooth movement agreed with the results of previous studies. ${ }^{4-7}$

Our results demonstrated a significant decrease in labial bone thickness at the crestal level during upper incisor retraction and a nonsignificant increase in labial bone thickness at midroot and apical area. These results are similar to the study done by Sarikaya et $\mathrm{al}^{8}$ and Ahn et al. ${ }^{9}$ The reason that the cervical area did not show an increase in alveolar bone on the labial side seems to be an inflammatory periodontal response concentrated in the cervical area, resulting in loss of alveolar bone in spite of the greater tensional force. Therefore, the entire alveolar housing, not merely the bone in the apical zone, should be considered when a clinician tries to define the therapeutic limits for orthodontic tooth movement. ${ }^{8}$

Wainwright ${ }^{5}$ histologically investigated the effects of faciolingual tooth movement as the root apex was moved through the cortical plate and then back into cancellous bone. He found that once the cortical plate had been penetrated, the buccal root surface became devoid of cortical bone. Although some osteogenesis took place during the 4-month retention period, it was insufficient to cover the root completely. The repair of perforation site took place only after the teeth relapsed. However, Ten Hoeve and Mulie $^{6}$ suggested on the basis of their laminographic evidence that the cortex would be reestablished within 6 months, no matter how extensive the tooth movement is. On the contrary, histologic studies have not indicated that the cortical plate is reestablished. In contrast to their findings, the present study did not show any newly formed thin cortical plate in the patients who developed perforation. In such conditions, use of the computed tomographic scan will help us to evaluate any repair of such perforation.

Duterloo $^{10}$ observed a definite shortening of the marginal aspect of the palatal cortex after orthodontic treatment but did not report any repair or remodeling 
even several years after treatment. Remmelink and van der Molen ${ }^{11}$ investigated Ten Hoeve and Mulie's ${ }^{6}$ patients several years after the original study and found welldefined dense cortical plate in association with relapse of torque of the anterior teeth. Perhaps, complete repair can take place only if relapse occurs, as Wainwright ${ }^{5}$ has suggested. Therefore, longitudinal studies are needed to investigate if repair of the alveolar bone defect takes place after space closure and during the retention period. In our study, there is a definite reduction of alveolar bone on the palatal side but we did not give time to allow osteoblastic activity to start the repair process; if any repair was to occur before, we did the T2 cephalogram. It would have been valuable to assess these same patients years from now to determine whether repair takes place.

Edwards ${ }^{12}$ studied a large group of people with class II malocclusion and bidental protrusion. He reported that despite prolonged palatal retraction and root torquing of incisors, the width of the anterior palate at the level of the apex remained unchanged. On the contrary, our results showed a much greater reduction in alveolar bone width at the coronal and midroot levels in maxilla than at the apical level, but in mandible, there is a greater reduction of alveolar bone width at the apical level. Based on these results, we suggest that marginal and midroot bone width is as important as apical width and that compensatory remodeling does not always match the amount of tooth movement.

In order to obtain appropriate alveolar bone response during incisor retraction in adult patients, we must pay attention to the fundamental knowledge to improve treatment strategy. For example, minor tooth movement and bone quantity limitation should be considered, force magnitude direction should be controlled, harmful effects of periodontal injury should be evaluated by regular radiographic examination, and ridge expansion osteotomy should be emphasized as an alternative way of decreasing the anatomical limitation of palatal portion. ${ }^{13}$

\section{CONCLUSION}

From the present study, it can be concluded that:

- When maxillary incisors are retracted, labial bone thickness at the midroot level (MxL2) and at apical level (MxL3) increased during upper incisor retraction.

- The change in maxillary labial bone thickness was not statistically significant except the width of the bone labial to the maxillary incisor decreased significantly at MxL1.
- There is a significant reduction in alveolar bone thickness on the lingual/palatal side after maxillary and mandibular incisor retraction.

- These results indicate that when maxillary and mandibular incisors are retracted, the risk of adverse effects may be present.

- The new alveolar bone formation may be expected after months, but the risks should be disclosed to the patients, and great care should be used when retracting maxillary and mandibular incisors. Very light forces and long-term activations to allow the alveolar bone to adapt may be useful in this regard.

\section{REFERENCES}

1. Reitan K. Effects of force magnitude and direction of tooth movement on different alveolar bone types. Angle Orthod 1964 Oct;34(4):244-255.

2. Handelman CS. The anterior alveolus: its importance in limiting orthodontic treatment and its influence on the occurrence of iatrogenic sequelae. Angle Orthod 1996;66(2):95-109.

3. DeAngelis V. Observations on the response of alveolar bone to orthodontic force. Am J Orthod 1970 Sep;58(3):284-294.

4. Vardimon AD, Oren E, Ben-Bassat Y. Cortical bone remodeling/ tooth movement ratio during maxillary incisor retraction with tip versus torque movements. Am J Orthod Dentofacial Orthop 1998 Nov;114(5):520-529.

5. Wainwright WM. Faciolingual tooth movement: its influence on the root and cortical plate. Am J Orthod 1973 Sep;64(3): 278-302.

6. Ten Hoeve A, Mulie RM. The effect of antero-postero incisor repositioning on the palatal cortex as studied with laminagraphy. J Clin Orthod 1976 Nov;10(11):804-822.

7. Wehrbein H, Bauer W, Diedrich P. Mandibular incisors, alveolar bone, and symphysis after orthodontic treatment. A retrospective study. Am J Orthod Dentofacial Orthop 1996 Sep;110(3):239-246.

8. Sarikaya S, Haydar B, Ciger S, Ariyurek M. Changes in alveolar bone thickness due to retraction of anterior teeth. Am J Orthod Dentofacial Orthop 2002 Jul;122(1):15-26.

9. Ahn HW, Moon SC, Baek SH. Morphometric evaluation of changes in the alveolar bone and roots of the maxillary anterior teeth before and after en masse retraction using cone- beam computed tomography. Angle Orthod 2013 Mar;83(2):212-221.

10. Duterloo HS. The impact of orthodontic treatment procedures on the remodelling of alveolar bone. Orthod Studieweek 1975;66:5-23.

11. Remmelink HJ, van der Molen AL. Effects of anteroposterior incisor repositioning on the root and cortical plate: a followup study. J Clin Orthod 1984 Jan;18(1):42-49.

12. Edwards JG. A study of the anterior portion of the palate as it relates to orthodontic therapy. Am J Orthod 1976 Mar;69(3):249-273.

13. Kim Y, Park JU, Kook YA. Alveolar bone loss around incisors in surgical skeletal Class III patients. Angle Orthod 2009 Jul;79(4):676-682. 